\title{
Cambios en la velocidad de crecimiento e indicadores nutricionales de Caiman yacare alimentados con diferentes dietas
}

\author{
Koza, G.A.; Barboza, N.N.; Fioranelli, S.A.; Mussart, N.B.; Coppo, J.A. \\ Cátedra de Fisiología, Facultad de Ciencias Veterinarias, UNNE, Sargento Cabral 2139, \\ Corrientes (3400), Argentina. Tel/fax 03783-425753. \\ E-mail: fisiologia@vet.unne.edu.ar.
}

\begin{abstract}
Resumen
Koza, G.A.; Barboza, N.N.; Fioranelli, S.A.; Mussart, N.B.; Coppo, J.A.: Cambios en la velocidad de crecimiento e indicadores nutricionales de Caiman yacare alimentados con diferentes dietas. Rev. vet. 21: 1, 28-33, 2010. La eficiencia de una dieta puede comprobarse mediante los aumentos del peso y dimensiones corporales, así como la optimización de indicadores bioquímicos nutricionales. El objetivo del trabajo fue comparar los efectos de dos diferentes dietas sobre el desarrollo de ejemplares sub-adultos de Caiman yacare en un criadero del nordeste argentino. Se emplearon 40 animales clínicamente sanos, de un año de edad. Inicialmente, los promedios de peso y longitud hocico-cloaca de los reptiles fueron $1,41 \pm 0,22 \mathrm{~kg}$ y $37,88 \pm 4,87 \mathrm{~cm}$. Aleatoriamente fueron divididos en dos lotes, alojándose en piletas separadas. Durante 105 días, ambos grupos consumieron una dieta base (70\% de la ración) consistente en pellets balanceados ( $88 \%$ de materia seca) con $30 \%$ de proteína bruta, $11 \%$ de extracto etéreo y $4,5 \%$ de cenizas totales. En la dieta A, el $30 \%$ restante de la ración fue carne vacuna sin hueso donde los parámetros antemencionados asumieron valores de $33,3 \%, 17,2 \%, 13,5 \%$ y $1,1 \%$. En la dieta B, el $30 \%$ restante fue carne de pollo con hueso $(37,6 \%, 18,4 \%, 11,6 \%$ y $7,7 \%$ respectivamente). Se realizaron pesajes, mediciones y estudios hemáticos al inicio y al final del ensayo. Se empleó un diseño completamente aleatorizado y se realizó análisis de la covarianza (ANCOVA). La dieta A, que contenía mayor proporción de grasas, produjo elevaciones significativas $(p<0,05)$ de triglicéridos, magnesio y potasio. La dieta $\mathrm{B}$, que contenía mayor proporción de proteínas y minerales, causó aumentos significativos del peso vivo, longitud hocico-cloaca, longitud de cabeza, concentración de hemoglobina corpuscular media, sodio, colesterol total, C-HDL y C-LDL. Se concluye que los escasos cambios registrados autorizan el uso de ambas dietas, pudiendo preferenciarse la que brinde mayores beneficios económicos.
\end{abstract}

Palabras clave: Caiman yacare, alimentación, desarrollo corporal, indicadores nutricionales.

\begin{abstract}
Koza, G.A.; Barboza, N.N.; Fioranelli, S.A.; Mussart, N.B.; Coppo, J.A.: Changes in growth rate and nutritional indicators of Cayman yacare fed on different diets. Rev. vet. 21: 1, 28-33, 2010. The efficiency of a diet can be evaluated by means of liveweight and corporal dimension increments, as well as the optimization of nutritional biochemical indicators. The objective of this trial was to compare the effects of two different diets on the growth of C. yacare sub-adult specimens, housed in a hatchery from northeastern Argentina. Forty clinically healthy yearling animals were used. Reptiles liveweight and length from muzzle to anus averages were initially $1.41 \pm 0.22 \mathrm{~kg}$ y $37.88 \pm 4.87 \mathrm{~cm}$. Animals were divided aleatorily into two groups and housed in separate tanks. During 105 days, both groups consumed a basal diet ( $70 \%$ of the ration) consistent in balanced pellets ( $88 \%$ of dry matter) with $30 \%$ of crude protein, $11 \%$ of ether extract and $4.5 \%$ of total ashes. For diet A, remaining $30 \%$ of the ration was beef without bone, in which values of parameters mentioned above were $33.3 \%, 17.2 \%, 13.5 \%$ and $1.1 \%$, respectively. For diet B, remaining $30 \%$ was chicken meat with bone $(37.6 \%, 18.4 \%, 11.6 \%$ and $7.7 \%$, respectively). Weight, size, and blood analysis were carried out at the beginning and the end of the assay. A totally randomized design and a statistical covariance analysis (ANCOVA) were applied for the statistic analysis. Diet $\mathrm{A}$, which contained a higher proportion of fat, produced significant increments $(\mathrm{p}<0.05)$ of triglycerides, magnesium and potassium. Diet $\mathrm{B}$, with a higher proportion of proteins and minerals, caused significant increments of liveweight, length from muzzle to anus, length of
\end{abstract}


head, mean corpuscular hemoglobin concentration, sodium, total cholesterol, HDL-C and LDL-C. It can be concluded that the minimum changes registered in this assay suggest the use of both diets indistinctly. When considering selection of one of them, economic factors should be taken into account.

Palabras clave: Caiman yacare, feeding, body growth, nutritional indicators.

\section{INTRODUCCIÓN}

En el nordeste argentino se lleva a cabo la cría de yacarés autóctonos con miras al aprovechamiento de su cuero y carne. El sistema empleado es la cosecha de huevos y ulterior crianza en cautiverio (ranching), con restitución de ejemplares juveniles al medio en cantidad semejante al porcentaje de individuos que hubieran sobrevivido en condiciones naturales, en salvaguarda del equilibrio ecológico y la conservación de las especies ${ }^{2}$. El sistema ranching y las medidas de control de la fauna silvestre han estimulado el aumento de la densidad poblacional de yacarés ${ }^{32}$.

A partir de la exportación de carne, cuero y esencias, esta producción genera divisas del orden de $8 \mathrm{mi}$ llones de dólares por año ${ }^{16}$. Indudablemente el cuero es el producto más valioso de los caimanes ${ }^{19}$, aunque el valor de la carne de estos reptiles no es despreciable, ya sea fresca o manufacturada en diferentes presentaciones ${ }^{26}$.

Para sustentar la ecuación productiva, entre otras medidas es menester adoptar un sistema de alimentación que genere rápido crecimiento a bajo costo, sin menoscabar la salud del animal. Numerosos grupos de trabajo persiguen este objetivo en el mundo entero ${ }^{1,11}$, $12,20,22-24,27,29$. Los incrementos del peso y las dimensiones corporales, así como la mejoría de los indicadores bioquímicos de estado nutricional, son algunas de las pautas que señalan la eficiencia de un plan de alimentación ${ }^{6}$. Los caimanes son carnívoros no selectivos que pueden consumir animales vivos o carroña ${ }^{13}$; en los criaderos su dieta se basa principalmente en alimentos balanceados comerciales, no siempre concebidos para reptiles ${ }^{24}$.

Todos los crocodílidos registran una alta asimilación de proteínas de origen animal, frente al consumo de proteínas vegetales ${ }^{11}$. Experimentando la adición de salvado de soja (a niveles de $0 ; 4,5 ; 9 ; 13,5$ y $18 \%$ ) en 100 ejemplares juveniles de $C$. yacare de 1 a 140 días de edad, se comprobó que a medida que se incrementaba el nivel del componente vegetal, se reducía el consumo de alimento. Si bien no fue afectado el peso de los reptiles, el salvado de soja provocó una reducción lineal en la longitud total y longitud hocico-cloaca ${ }^{1}$.

Estudios sobre sus particularidades nutricionales indican que las dietas ricas en proteínas de origen animal producen efectos leves sobre el peso corporal, pero importantes sobre la longitud total ${ }^{23}$. Por otra parte, altos niveles de grasa en la dieta dificultan la digestión de proteínas ${ }^{9}$, a pesar de lo cual una fuente dietaria que contemple la ingesta de ácidos grasos esenciales sería importante para lograr mayor velocidad de crecimiento ${ }^{29}$. Los crocodílidos parecen incapaces de asimilar compuestos polisacáridos ${ }^{8}$.

La temperatura ambiental es vital en el comportamiento alimentario y digestivo de estos reptiles. La mayoría de los cocodrilos deja de alimentarse cuando la temperatura desciende por debajo de $25^{\circ} \mathrm{C}$, por lo cual su metabolismo disminuirá notablemente mientras avance el ayuno ${ }^{15,28}$, situación que puede provocar importantes variaciones de los componentes sanguíneos ${ }^{31}$. En anfibios y reptiles, la concentración de los componentes de la sangre sería poderosamente influenciada por sus peculiares características fisiológicas, tales como ectotermia, ayuno durante el letargo invernal y cambios metabólico-conductales relacionados a la temperatura exterior ${ }^{10}$.

El objetivo del trabajo fue comparar los efectos de dos diferentes dietas sobre el desarrollo de ejemplares sub-adultos de Caiman yacare en un criadero del nordeste argentino, mediante controles de peso, dimensiones corporales y parámetros hemáticos indicadores de estado nutricional.

\section{MATERIAL Y MÉTODOS}

La experiencia se realizó durante la temporada estival, desde diciembre de 2008 a marzo de 2009 (105 días) en un criadero de la localidad de Santa Ana, Provincia de Corrientes, Argentina. Se emplearon 40 ejemplares sub-adultos (un año de edad) de Caiman yacare clínicamente sanos. En promedio, el peso vivo inicial fue de $1,41 \pm 0,22 \mathrm{~kg}$ y y la longitud hocico-cloaca de $37,88 \pm 4,87 \mathrm{~cm}$. Los sujetos experimentales se dividieron en dos lotes de 20 animales cada uno $(50 \%$ de cada sexo), los cuales permanecieron alojados en piletas separadas.

La dieta base fue la misma en ambos grupos; constituyó el 70\% de la ración y consistió en un alimento balanceado cuya materia seca fue del $88 \%$, conteniendo $30 \%$ de proteína bruta, $11 \%$ de extracto etéreo y $4,5 \%$ de cenizas totales. En la dieta A, el 30\% restante de la ración fue un complemento de carne vacuna sin hueso (materia seca $33,3 \%$, proteína bruta $17,2 \%$, extracto etéreo $13,5 \%$ y ceniza $1,1 \%$ ). En la dieta B, el $30 \%$ restante de la ración fue un complemento de carne de pollo con hueso (materia seca $37,6 \%$, proteína bruta $18,4 \%$, extracto etéreo $11,6 \%$ y cenizas 7,7\%). La Tabla 1 posibilita una mejor visualización de los componentes de las dietas. 
Tabla 1. Composición centesimal del alimento.

\begin{tabular}{lccc}
\hline & $\begin{array}{c}\text { dieta base } \\
(70 \%)\end{array}$ & $\begin{array}{c}\text { compl. dieta A } \\
(30 \%)\end{array}$ & $\begin{array}{c}\text { compl. dieta B } \\
(30 \%)\end{array}$ \\
\hline MS \% & 88 & 33,3 & 37,6 \\
PB \% & 30 & 17,2 & 18,4 \\
EE \% & 11 & 13,5 & 11,6 \\
CT \% & 4,5 & 1,1 & 7,7 \\
\hline
\end{tabular}

MS: materia seca, PB: proteína bruta, EE: extracto etéreo, CT: cenizas totales, compl: complemento.
Todos los análisis bioquímicos estuvieron sujetos a un test de control de calidad, llevado a cabo con patrones de comparación liofilizados (Standatrol). Las fracciones proteicas (albúminas y globulinas alfa, beta y gamma) fueron separadas por electroforesis en acetato de celulosa (Cellogel, coloración amidoschwartz) y cuantificadas por densitometría a $610 \mathrm{~nm}$ en aparato Citocon CT-440, equipado con microprocesador para el cálculo de fracciones e impresora de ferogramas.

En el marco de un diseño completamente aleatoriza-

Tabla 2. Efecto de las dietas sobre el peso vivo $(\mathrm{kg})$ y las variables morfométricas $(\mathrm{cm})$.

\begin{tabular}{lcccccc}
\hline & $\mathrm{PV}$ & $\mathrm{LT}$ & $\mathrm{HC}$ & $\mathrm{PT}$ & $\mathrm{LC}$ & $\mathrm{AC}$ \\
\hline dieta A & $2,71 \pm 0,12$ & $79,69 \pm 2,18$ & $41,73 \pm 0,81$ & $23,27 \pm 0,58$ & $9,90 \pm 0,21$ & $6,64 \pm 0,13$ \\
dieta B & $3,08 \pm 0,12$ & $85,47 \pm 2,18$ & $44,20 \pm 0,81$ & $24,14 \pm 0,58$ & $10,51 \pm 0,21$ & $6,99 \pm 0,13$ \\
tratam. p & $0,04^{*}$ & 0,07 & $0,04^{*}$ & 0,30 & $0,04^{*}$ & 0,08 \\
ANCOVA F & 8,85 & 0,05 & 7,08 & 8,41 & 2,11 & 9,61 \\
ANCOVA p & 0,008 & 0,81 & 0,02 & 0,01 & 0,16 & 0,007 \\
\hline
\end{tabular}

Promedios finales \pm error estándar, PV: peso vivo, LT: longitud total, HC: longitud hocico-cloaca, PT: perímetro torácico, LC: longitud cabeza, AC: ancho cabeza, tratam.: tratamiento, ANCOVA: análisis de covarianza, F: valor de F, p: significancia $(<0,05)$, *diferencias significativas.

Los caimanes de ambos grupos fueron alimentados ad-libitum de lunes a viernes, con una oferta equivalente al $25 \%$ del peso vivo promedio. Al inicio y al final de la experiencia se realizaron pesajes en una balanza, mediciones corporales con cinta métrica metálica (longitud hocico-cloaca, longitud total, longitud de cabeza, ancho de cabeza, perímetro torácico) y extracciones de sangre con jeringa y aguja, a partir del seno venoso occipital. Una alícuota fue utilizada para obtener suero y la otra fue tratada con anticoagulante (EDTA) para efectuar determinaciones de hematocrito (centrifugación de capilares a $12.000 \mathrm{rpm}$ ) y hemoglobina (fotocolorimetría a $540 \mathrm{~nm}$ en aparato L.Mannheim 4010, técnica de la cian-meta-Hb, reactivos Wiener Lab), previa separación de los núcleos libres de los eritrocitos hemolizados, como se recomienda para reptiles ${ }^{3}$ . La concentración de hemoglobina corpuscular media (CHCM) se obtuvo por cálculo convencional.

Con el suero sanguíneo se realizaron determinaciones bioquímicas en un espectrofotómetro Zeltec ZL5000 UV-visible, con reactivos Wiener Lab, a saber: glucosa (método de la oxidasa-peroxidasa, evaluada a $505 \mathrm{~nm}$ ), fructosamina (tetrazolio, $530 \mathrm{~nm}$ ), proteínas totales (biuret, $540 \mathrm{~nm}$ ), urea (ureasa, $570 \mathrm{~nm}$ ), creatinina (picrato alcalino, $520 \mathrm{~nm}$ ), calcio (cresolftaleincomplexona, $570 \mathrm{~nm}$ ), fósforo inorgánico (fosfomolibdato, $620 \mathrm{~nm}$ ), magnesio (azul de xilidilo, $510 \mathrm{~nm}$ ), colesterol total (colesterol-oxidasa, $505 \mathrm{~nm}$ ), triglicéridos (lipasaperoxidasa, $505 \mathrm{~nm}$ ), colesterol ligado a lipoproteínas de alta densidad (CHDL) y baja densidad (CLDL) por precipitación selectiva de las lipoproteínas (fosfotungstato y polivinilo respectivamente), con ulterior valoración fotométrica por la técnica enzimática citada. Sodio y potasio se evaluaron en un fotómetro de llama Metrolab 305-D (a 586 y $768 \mathrm{~nm}$ respectivamente, reactivos Biopur).
Tabla 3. Diferencias de peso vivo y longitud, según dieta.

\begin{tabular}{lcccc}
\hline & \multicolumn{2}{c}{ peso vivo $(\mathrm{kg})$} & \multicolumn{2}{c}{ hocico-cloaca $(\mathrm{cm})$} \\
& dieta A & dieta B & dieta A & dieta B \\
\hline$\overline{\mathbf{X}}$ inicial (día 0) & 1,45 & 1,38 & 37,67 & 38,10 \\
$\overline{\mathbf{X}}$ final (día 105) & 2,71 & 3,08 & 41,73 & 44,20 \\
ganancia total & 1,26 & 1,70 & 4,06 & 6,10 \\
ganancia diaria & 0,012 & 0,016 & 0,038 & 0,058 \\
diferencia A - B & \multicolumn{2}{c}{ significativa } & significativa \\
\hline
\end{tabular}

do, se realizó análisis de la covarianza (ANCOVA) utilizando como covariables los valores iniciales de cada variable dependiente, a efectos de controlar el error y aumentar la precisión. Los cálculos se efectuaron con el auxilio del programa Statistica 6.0, 2002. Para todas las inferencias se estableció un nivel de significancia del $5 \%$, por debajo del cual fue rechazada la hipótesis nula de igualdad.

\section{RESULTADOS Y DISCUSIÓN}

La Tabla 2 indica que la dieta B provocó aumentos estadísticamente significativos sobre el peso vivo, longitud hocico-cloaca y longitud de cabeza. En cambio, ninguna de las variables dependientes se incrementó con la dieta A. En los reptiles alimentados con la dieta B también aumentaron de manera no significativa las restantes variables morfométricas, a saber: perímetro torácico, ancho de cabeza y longitud total, en los dos últimos casos con significancias muy cercanas al nivel de rechazo de la hipótesis nula $(0,08$ y 0,07 respectivamente). Dado que la punta de la cola del caimán es frágil y frecuentemente se quiebra en los criaderos, en términos prácticos la longitud hocico-cloaca (significativa en el presente estudio) resulta más confiable que 
Tabla 4. Efecto de las dietas sobre el proteinograma (g/dl).

\begin{tabular}{lccccccc}
\hline & $\mathrm{PT}$ & $\mathrm{AL}$ & $\mathrm{GT}$ & $\mathrm{GA}$ & $\mathrm{GB}$ & $\mathrm{GG}$ & $\mathrm{AG}$ \\
\hline dieta A & $4,95 \pm 0,09$ & $1,37 \pm 0,06$ & $3,56 \pm 0,11$ & $0,61 \pm 0,03$ & $1,08 \pm 0,02$ & $1,85 \pm 0,07$ & $0,39 \pm 0,02$ \\
dieta B & $5,01 \pm 0,09$ & $1,34 \pm 0,07$ & $3,69 \pm 0,13$ & $0,58 \pm 0,04$ & $1,08 \pm 0,03$ & $2,05 \pm 0,07$ & $0,36 \pm 0,03$ \\
tratam. p & 0,61 & 0,79 & 0,45 & 0,64 & 0,96 & 0,07 & 0,49 \\
ANCOVA F & 1,26 & 0,30 & 0,003 & 0,001 & 6,11 & 1,12 & 0,07 \\
ANCOVA p & 0,28 & 0,59 & 0,95 & 0,99 & 0,03 & 0,31 & 0,79 \\
\hline
\end{tabular}

Promedios finales \pm error estándar, PT: proteínas totales, AL: albúminas, GT: globulinas totales, GA: globulinas alfa, GB: globulinas beta, GG: globulinas gamma, AG: relación albúminas /globulinas, tratam.: tratamiento, ANCOVA: análisis de covarianza, F: valor de F, p: significancia $(<0,05)$.

Tabla 5. Efecto de las dietas sobre el hemograma y algunos metabolitos séricos.

\begin{tabular}{lccccccc}
\hline & HT & HB & CH & GL & FR & UR & CR \\
\hline dieta A & $20 \pm 0,64$ & $5,78 \pm 0,24$ & $29 \pm 0,64$ & $1,16 \pm 0,07$ & $149 \pm 12,1$ & $0,11 \pm 0,003$ & $6,99 \pm 0,47$ \\
dieta B & $19 \pm 0,65$ & $6,08 \pm 0,24$ & $32 \pm 0,62$ & $1,17 \pm 0,08$ & $176 \pm 12,0$ & $0,11 \pm 0,003$ & $6,21 \pm 0,48$ \\
tratam. p & 0,34 & 0,38 & $0,009 *$ & 0,96 & 0,14 & 0,84 & 0,27 \\
ANCOVA F & 2,74 & 2,98 & 0,13 & 4,64 & 0,31 & 3,20 & 0,79 \\
ANCOVA p & 0,11 & 0,10 & 0,72 & 0,04 & 0,58 & 0,09 & 0,38 \\
\hline
\end{tabular}

Promedios finales \pm error estándar, HT: hematocrito (\%), HB: hemoglobina (g/dl), CH: concentración de hemoglobina corpuscular media (\%), GL: glucosa (g/l), FR: fructosamina (umol/l), UR: urea (g/l), CR: creatinina (mg/l), tratam.: tratamiento, ANCOVA: análisis de covarianza, F: valor de F, p: significancia $(<0,05)$, *diferencias significativas.

la longitud total (no significativa) para valorar el crecimiento ${ }^{20}$. No quedan dudas que la velocidad de crecimiento, en términos del peso vivo y las dimensiones corporales, fue favorecida por la dieta B.

En la Tabla 3 se amplifica la evolución de las dos variables más importantes para estimar el desarrollo corporal de los reptiles: el peso vivo y la longitud hocicocloaca ${ }^{18}$. Se aprecia que en este ensayo, la velocidad de crecimiento de C. yacare entre los 12 y los $15 / 16$ meses de edad fue mayor en los ejemplares alimentados con la dieta $\mathrm{B}$, grupo en el cual los aumentos de peso vivo se situaron en $16 \mathrm{~g} /$ día (versus $12 \mathrm{~g}$ /día para la dieta $\mathrm{A}$ ) y la longitud hocico-cloaca creció $0,58 \mathrm{~mm} /$ día con la dieta $\mathrm{B}$ (versus $0,38 \mathrm{~mm} /$ día con la dieta $\mathrm{A}$ ).

Las dietas ensayadas produjeron velocidades de crecimiento similares o menores a las obtenidas por otros investigadores en diferentes especies de caimanes. Así, ejemplares neonatos de Caiman crocodilus crocodilus ("baba venezolana") revelaron a los 10 meses ritmos de crecimiento longitudinal de 1,0 a $3,9 \mathrm{~mm} /$ día ${ }^{20}$. Para la misma especie, otros autores registraron velocidades más reducidas $(0,5 \mathrm{~mm} /$ día) probablemente por la dieta suministrada ${ }^{18}$. Entre los 3 y 11 primeros meses de vida la tasa de crecimiento de Crocodylus intermedius (cocodrilo del Orinoco) osciló de 2,6 a 3,0 $\mathrm{mm} / \mathrm{día}(\overline{\mathrm{x}}=2,78 \mathrm{~mm} / \mathrm{día})^{21}$. Neonatos y juveniles de Crocodylus acutus (cocodrilo americano, Florida) crecieron a razón de $1,04 \mathrm{~mm} / \mathrm{dí}^{25}$, aunque otros autores registraron $0,93 \mathrm{~mm} /$ día $^{7}$. Melanosuchis niger (caimán del Amazonas) exhibió un crecimiento de 0,62 $\mathrm{mm} / \mathrm{día}$ ${ }^{7}$, similar al aquí registrado en C. yacare alimentados con la dieta B.

Los resultados obtenidos en el presente ensayo son coincidentes con trabajos de investigadores que demostraron que la carne blanca (pollo, pescado) puede ser tan adecuada como la roja (vaca, cerdo) para la alimentación de los caimanes. En efecto, controlando durante 7 meses el crecimiento a través del aumento del peso y la variación de los parámetros morfométricos, ejemplares juveniles de Caiman latirostris fueron sometidos a dietas basadas en pollo, cerdo, pescado y una mezcla de las tres anteriores. Todas las dietas lograron aumentos de peso y dimensiones, sin diferencias significativas entre ellas ${ }^{23}$.

Los valores hematológicos y bioquímicos obtenidos en esta experiencia enmarcaron en el intervalo de referencia publicado para C. yacare ${ }^{4}$. La Tabla 4 señala que ninguna de las dos dietas modificó significativamente las variables del proteinograma. Se advirtió una leve tendencia al aumento de proteínas totales, globulinas totales y gamma globulinas en los animales sometidos a la dieta B, y de albúminas, globulinas alfa y relación albúminas /globulinas en los ejemplares asignados a la dieta A. En los mamíferos, es dable aguardar la elevación de las proteínas plasmáticas, especialmente albúminas, ante el aumento de la carga proteica de la dieta ${ }^{5}$, circunstancia ausente en este ensayo quizás porque las tasas proteicas de las dietas no eran tan diferentes. Desde otro punto de vista, es auspicioso que ninguna de las dietas causara elevación de alfa globulinas (inflamación aguda), beta globulinas (sobrecarga hepática) ni gamma globulinas (hipersensibilidad hacia algún componente de la dieta) ${ }^{6}$.

En la Tabla 5 puede advertirse que, a excepción de la concentración de hemoglobina corpuscular media, los valores de hematocrito, hemoglobina, glucosa, fructosamina, urea y creatinina no registraron diferencias significativas entre las dietas A y B. Ello podría señalar que ambos sistemas de alimentación fueron eficaces para sostener los parámetros de la serie roja san- 
Tabla 6. Efecto de las dietas sobre el lipidograma (g/l).

\begin{tabular}{lcccc}
\hline & $\mathrm{CT}$ & $\mathrm{TG}$ & $\mathrm{C}-\mathrm{HDL}$ & $\mathrm{C}-\mathrm{LDL}$ \\
\hline dieta A & $1,12 \pm 0,06$ & $1,98 \pm 0,20$ & $0,16 \pm 0,03$ & $0,41 \pm 0,05$ \\
dieta B & $1,53 \pm 0,07$ & $0,68 \pm 0,21$ & $0,32 \pm 0,02$ & $0,67 \pm 0,06$ \\
tratam. p & $0,0005^{*}$ & $0,0003^{*}$ & $0,004^{*}$ & $0,006^{*}$ \\
ANCOVA F & 2,31 & 0,26 & 0,03 & 0,43 \\
ANCOVA p & 0,14 & 0,61 & 0,85 & 0,52 \\
\hline
\end{tabular}

Promedios finales \pm error estándar, CT: colesterol total, TG: triglicéridos, C-HDL y C-LDL: colesterol ligado a lipoproteínas de alta y baja densidad respectivamente, tratam.: tratamiento, ANCOVA: análisis de covarianza, F: valor de F, p: significancia $(<0,05)$, *diferencias significativas.

Tabla 7. Efecto de las dietas sobre el ionograma.

\begin{tabular}{lccccc}
\hline & $\mathrm{Na}$ & $\mathrm{K}$ & $\mathrm{Ca}$ & $\mathrm{P}$ & $\mathrm{Mg}$ \\
\hline dieta A & $145 \pm 1,83$ & $5,01 \pm 0,23$ & $9,66 \pm 0,17$ & $5,06 \pm 0,26$ & $3,10 \pm 0,13$ \\
dieta B & $148 \pm 1,84$ & $4,07 \pm 0,23$ & $9,37 \pm 0,18$ & $4,84 \pm 0,27$ & $2,58 \pm 0,14$ \\
tratam. p & 0,21 & $0,01^{*}$ & 0,26 & 0,56 & $0,01^{*}$ \\
ANCOVA F & 0,36 & 2,60 & 0,04 & 0,39 & 1,98 \\
ANCOVA p & 0,56 & 0,12 & 0,84 & 0,54 & 0,18 \\
\hline
\end{tabular}

Promedios finales \pm error estándar, Na: sodio (meq/l), K: potasio (meq/l), Ca: calcio total (mg/dl), P: fósforo inorgánico (mg/dl), Mg: magnesio (mg/ dl), tratam.: tratamiento, ANCOVA: análisis de covarianza, F: valor de F, p: significancia $(<0,05)$, *diferencias significativas.

guínea, así como la homeostasis de los metabolismos glucídico y nitrogenado ${ }^{5}$. La fructosamina, indicador retrospectivo del metabolismo hidrocarbonado, aunque de manera no significativa se elevó moderadamente en los animales sometidos a la dieta B, señalando ${ }^{6}$ que las glucemias de este grupo debieron ser más altas que las de los reptiles asignados a la dieta $\mathrm{A}$.

La Tabla 6 muestra que los ejemplares de C. yacare alimentados con la dieta $\mathrm{B}$ culminaron la experiencia con valores plasmáticos de colesterol total significativamente más elevados que los sometidos a la dieta A. Ello, sumado al hecho que esta especie parece responder al patrón metabólico lipoproteico tipo "LDL" (C-LDL > $\mathrm{C}-\mathrm{HDL}$ ), permite inferir que los animales alimentados con la dieta $\mathrm{B}$ ostentaron una mayor susceptibilidad al riesgo aterogénico ${ }^{6}$. En cambio, los triglicéridos resultaron significativamente más altos en los animales que consumieron la dieta $\mathrm{A}$, en coincidencia con su mayor contenido de grasas ${ }^{5}$.

La Tabla 7 indica que la concentración plasmática de casi todos los minerales fue más alta en los animales que consumieron la dieta $\mathrm{A}$, significativamente para magnesio y potasio, mientras que para calcio total y fósforo inorgánico solo fue una tendencia. En cambio, el sodio fue ligeramente menor en A que en B. Dado que ningún electrolito se apartó de su intervalo de referencia ${ }^{4}$, puede aseverarse que ambas dietas aseguraron el mantenimiento de la osmolalidad del medio interno, la polarización de membranas, la estructura de huesos y dientes y otras importantes funciones.

Cabe destacar que el presente ensayo se realizó íntegramente en estación cálida, por lo cual se marginó del diseño experimental el efecto del cese de alimenta- ción propio del letargo invernal ${ }^{10,13}, \mathrm{y}$ por ende la disminución plasmática de algunos indicadores nutricionales y la variación de ciertos parámetros metabólicos ${ }^{4}$. Son escasas las publicaciones que relacionan la velocidad de crecimiento y/o el alimento consumido, con las fluctuaciones del medio interno de estos reptiles. Los valores hematológicos, además de ser afectados por la alimentación, varían según el ambiente, la temperatura y el sistema de manejo ${ }^{30}$. Se asevera que el crecimiento de los caimanes es intermitente y estrechamente dependiente de la temperatura ambiental ${ }^{14}$.

Algunos autores consideran que los crocodílidos deberían ser alimentados con dietas de alto contenido en proteínas y grasas; alrededor del $25-30 \%$ de la energía metabolizable debería provenir de las primeras y del $30-60 \%$ de las segundas. Así, para lograr un mejor crecimiento en Alligator mississippiensis, se descubrió que el consumo de proteínas debe ser de alrededor del $42 \%$ de la energía metabolizable ${ }^{17}$, tasa superior a la empleada en el presente ensayo.

Ejemplares juveniles de $C$. crocodilus crocodilus fueron divididos en grupos y alimentados con tres diferentes dietas a base de pescado, pollo y vísceras de ganado vacuno (pulmón y corazón). La mayor tasa de crecimiento en longitud y peso se obtuvo en los animales sometidos a la tercera dieta ${ }^{20}$, cuya tasa de proteínas era similar a la empleada en la presente experiencia.

En un trabajo anterior se compararon cuatro dietas (pescado, crustáceos, moluscos e insectos acuáticos) en 20 ejemplares de Caiman crocodilus yacare desde su nacimiento hasta los 88 días de vida. Ninguna de las monodietas ensayadas resultaron adecuadas para esta especie, aunque la mayor tasa de crecimiento se obtuvo con pescados ${ }^{27}$. Neonatos de Caiman crocodilus fuscus fueron sometidos a diferentes dietas (pollos de desecho, caseína, harina integral de gallina y harina de pescado), demostrándose que la más alta velocidad de crecimiento (mayores ganancias de peso y aumentos de longitud total) ocurrieron en el lote alimentado con harina de pescado ${ }^{12}$. En otro ensayo, 90 neonatos de C. acutus fueron alimentados con tres dietas experimentales (A: pescados marinos, hígado y pulmón de res; B: pescado y C: larvas de moscas), resultando más efectiva la dieta $\mathrm{B}$ (pescado) que las dietas $\mathrm{A}$ y $\mathrm{C}^{22}$. A partir de estas publicaciones quedaría evidenciada una mayor eficiencia nutricional del pescado sobre otras dietas, aunque debe tenerse en cuenta que el pescado fue 14 veces más caro que el resto de los componentes ${ }^{22}$.

En conclusión, los escasos cambios registrados tornan dificultosa la tarea de señalar cuál es la dieta más ventajosa para aumentar la velocidad de crecimiento de C. yacare. Prima facie, surge que la dieta B resulta li- 
geramente más eficiente al acelerar el desarrollo en términos de peso vivo y longitud corporal, pero las cifras no implican diferencias contundentes capaces de modificar considerablemente la duración del lapso de crianza. La similitud de los resultados obtenidos habilita el empleo de ambas dietas, con lo cual emergería como ventajosa la circunstancia de poder preferenciar la que el mercado ofrezca en ese momento a menor costo.

\section{REFERENCIAS}

1. Aleixo VM, Cotta T, Logato PV, Gomes AI, Fialho ET. 2002. Efeitos da adiçao de diferentes teores de farelo de soja na dieta sobre o desenvolvimento de filhotes de jacare do pantanal (Caiman yacare). Cienc Agrotéc 26: 411-417.

2. Boló Bolaño E, Stamatti G, Parera A, Prado WS, Moreno D. 2000. Conservation and sustainable use of the broad snouted caiman (Caiman latirostris) and yacaré (Caiman yacare) in Argentina. Anales XV Reunión Grupo Esp Cocodr (Varadero, Cuba), p. 27.

3. Campbell TW. 1996. Clinical Pathology. In: Reptile Medicine and Surgery (Mader DR Ed.), Saunders, Philadelphia, p. 248-257.

4. Coppo JA, Mussart NB, Barboza NN, Fioranelli SA, Koza GA, Prado WR. 2006. Physiological changes in serum glucidic and nitrogenic analytes from captive argentine authoctonous caimans. Rev Vet 17: 103-108.

5. Coppo JA. 2008. Fisiología comparada del medio interno, Eucasa, Salta, 310 p.

6. Coppo JA. 2010. Interpretación de análisis clínicos en perros y gatos, Ed. Eucasa, Salta (Argentina), 370 p.

7. Dowling HG, Brazaitis P. 1966. Size and growth in captive crocodilians. Int Zoo Yearbook 6: 256-270.

8. Fraser C. 1988. Manual Merck de veterinaria, $3^{\circ}$ ed., Centrum Ed., Buenos Aires, 2092 p.

9. Garnett S. 1988. Digestion, assimilation and metabolism of captive estuarine crocodiles, Crocodylus porosus. Comp Biochem Physiol A 90: 23-29.

10. Goldstein L. 1982. Fisiología comparada, Ed. Interamericana, México, $454 \mathrm{p}$.

11. Herbert JD, Coulson RA. 1975. Free amino acids in crocodilians fed proteins of different biological value. $J$ Nutr 105: 616-623.

12. Jiménez G, Afanador G, Viorello HJ. 2006. Evaluación de la harina de carne de gallina en alimentos balanceados para babilla (Caiman crocodilus). Tesis, Universidad Nacional de Colombia, Bogotá, $74 \mathrm{p}$.

13. Kardong KV. 1998. Vertebrates: comparative anatomy, function, evolution, $2^{\circ}$ ed., McGraw Hill, New York, 747 p.

14. Lance VA. 2003. Alligator physiology and life history: the importance of temperature. Exp Geront 38: 801-805.

15. Lane TJ. 1996. Crocodilians. In: Reptile Medicine and Surgery (Mader DR Ed.), Saunders, Philadelphia, p. 78-98.

16. Larriera A. 1997. Programa de ranching experimental en Argentina. Anales III Congr Internac Manejo Fauna Silv (Santa Cruz, Bolivia), p. 166.
17. Mader DR. 1996. Reptile medicine and surgery, Saunders, Philadelphia, $415 \mathrm{p}$.

18. Magnusson W, Sanaiotti T. 1995. Growth of Caiman crocodilus crocodilus in central amazonia, Brazil. Copei 2: 498-501.

19. Micucci PA, Waller T. 1995. Los yacarés en Argentina: hacia un aprovechamiento sustentable, Ed. Fund Banco Bica, Santa Fe (Argentina), p. 81-112.

20. Pérez AT. 2000. Crecimiento de Caiman crocodilus en cautiverio. Interciencia 25: 442-446.

21. Pérez AT, Rodríguez JC. 2005. Influencia de la temperatura del aire y del agua en el crecimiento de Crocodylus intermedius. Boletín Centro Investig Biol (Caracas) 39: 25-26.

22. Pérez Gómez M, Naranjo C, Reyes B, Vega I. 2009. Influencia de dos tipos de dietas sobre la talla y el peso corporal de Crocodylus acutus. Acta Zool Mex 25: 151-160.

23. Pinheiro MS, Lavorenti A. 2001. Growth of broad-nosed caiman Caiman latirostris hatchlings, fed with diets of animal origin. Braz J Biol 61: 421-429.

24. Prado WS, Gómez O, Balla P. 2001. Crianza en cautiverio y reintroducción de individuos (C. latirostris y C. yacare). Bol Técn Fund Vida Silv Arg 55: 26-38.

25. Rodríguez E, Rodríguez MA. 1989. Evaluación del crecimiento y levante de neonatos y juveniles de Crocodylus acutus. Trianea 3: 53-60.

26. Romanelli PF, Caseri R, Lopes-Filho JF. 2002. Meat processing of pantanal alligator (Caiman crocodilus yacare). Cienc Tecnol Aliment 22: 70-75.

27. Santos SA, Pinheiro MS, Silva RA. 1993. Efeitos de diferentes dietas naturais no desenvolvimento inicial de Caiman crocodilus yacare. Rev Bras Zoot 22: 406-412.

28. Schoeb TR, Heaton TG, Clemmons RM, Carbonneau DA, Woodward AR, Shelton D, Poppenga RH. 2002. Clinical and necropsy findings associated with increased mortality among American alligators of Lake Griffin, Florida. J Wildl Dis 38: 320-337.

29. Staton MA, Edwards HM, Brisbin IL, Joanen T, McNease L. 1990. Protein and energy relationships in the diet of Alligator mississippiensis. J Nutr 120: 775-785.

30. Swanepoel D, Boomker J, Kriek NP. 2000. Selected chemical parameters in the blood and metals in the organs of Crocodylus niloticus. Onderstepoort J Vet Res 67: 141148.

31. Uhart M, Prado W, Beldoménico P, Rossetti C, Ferreyra MC, Martínez A, Bardón JC, Avilés G, Karesh W. 2001. Estudios sanitarios comparativos de yacarés (Caiman latirostris y Caiman yacare) silvestres y cautivos. Bol Técn Fund Vida Silv Arg 55: 39-50.

32. Waller T, Micucci PA. 1993. Relevamiento de la distribución, hábitat y abundancia de los crocodilios de la Provincia de Corrientes, Argentina. Memorias 1ra Reunión Reg Grupo Esp Cocodr (Santa Marta, Colombia), p. 341-385. 